\title{
ZEIGT HER EURE ...
}

\section{Liebe Kolleginnen, liebe Kollegen!}

In der heutigen Zeit ist die virtuelle Visitenkarte weitaus wichtiger als das gedruckte Wort. Unser Fußabdruck muss im www gut ersichtlich sein und wir müssen die sozialen Medien für uns nutzen können, um als Akupunkturgesellschaft auf uns aufmerksam zu machen.

Obwohl die beste Werbung nach wie vor die Mundpropaganda ist und nichts wichtiger als zufriedene KollegInnen ist, müssen wir doch Facebook, Instagram und Co zu nutzen wissen, um auch junge Kolleginnen und Kollegen mit unserem Ausbildungsangebot zu erreichen.

Die Basis für einen guten virtuellen Auftritt ist und bleibt die Homepage. So haben wir mit viel Herzblut und kreativem Input unsere Website komplett erneuert. Auf das Ergebnis bin ich wirklich stolz und würde Sie gern zu einem Besuch einladen: www.ogka.at

Da merkt man nochmals ganz deutlich, wie breit unser Angebot doch aufgestellt ist.Von den drei Basissäulen Körperakupunktur, TCM und Auriculomedizin ausgehend, haben wir mittlerweile 12 Spezialausbildungen als Weiterbildungsoption anzubieten.

Zudem wenden wir uns durch die Vorlesung an der Medizinischen Universität Graz gezielt an die Medizin-
studentInnen als potenziellen Akupunkturnachwuchs. Wir bieten neuerdings auch eine Akupunkturausbildung für Hebammen an, diese haben wir ganz speziell auf die Ansprüche dieser wichtigen Berufsgruppe zugeschnitten.

Neben den Ausbildungen gewinnt unsere Homepage natürlich mit unserer umfangreichen Studiendatenbank, in der über 600 Akupunkturstudien - monatlich aktualisiert $-\mathrm{zu}$ finden sind.

Neu ist eine Job- und Gebrauchtgerätebörse, um unseren Mitgliedern bestmöglichen Service bieten zu können.

Ich bin unglaublich stolz auf dieses virtuelle Baby, das wir geschaffen haben und bin zuversichtlich, dass es eine gute Plattform für die Verbreitung der Akupunktur ist.

In diesem Sinne!

Like us on facebook and Instagram (;)

Ihre

O. Pojer 